\title{
The Pharmacokinetic Effect of Itraconazole and Voriconazole on Ripretinib in Beagle Dogs by UPLC-MS/MS Technique
}

\author{
Hui-jun Wang' \\ Chun-yan Zhou' \\ Yan-ding Su' \\ Kai-feng Gou' \\ Xiao-nan Geng' \\ Xiang-jun Qiu (iD) ${ }^{1,2}$ \\ 'Department of Pharmacy, School of \\ Basic Medical Sciences, Henan University \\ of Science and Technology, Luoyang, \\ 47I 023, People's Republic of China; \\ ${ }^{2}$ Functional Experiment Teaching Center, \\ School of Basic Medical Sciences, Henan \\ University of Science and Technology, \\ Luoyang, 47I023, People's Republic of \\ China
}

Background: A new UPLC-MS/MS technique for the determination of ripretinib in beagle dog plasma was developed, and the pharmacokinetic effects of voriconazole and itraconazole on ripretinib in beagle dogs were studied.

Methods: After extraction with ethyl acetate under alkaline conditions, ripretinib was detected using avapritinib as the internal standard (IS). The mobile phases were $0.1 \%$ formic acid-acetonitrile. The scanning method was multi-reaction monitoring using ESI+ source, and the ion pairs for ripretinib and IS were $\mathrm{m} / \mathrm{z} 509.93 \rightarrow 416.85$ and $499.1 \rightarrow 482.09$, respectively. This animal experiment adopted a three period self-control experimental design. In the first period, ripretinib was orally administered to six beagle dogs at a dose of $5 \mathrm{mg} / \mathrm{kg}$. In the second period, the same six beagle dogs were orally given itraconazole at a dose of $7 \mathrm{mg} / \mathrm{kg}$, after $30 \mathrm{~min}$, ripretinib was orally given. In the third period, voriconazole at a dose of $7 \mathrm{mg} / \mathrm{kg}$ was given orally, and then ripretinib was orally given. At different time points, the blood samples were collected. The concentration of ripretinib was detected, and the pharmacokinetic parameters of ripretinib were calculated.

Results: Ripretinib had a good linear relationship in the range of $1-1000 \mathrm{ng} / \mathrm{mL}$. The precision, accuracy, recovery, matrix effect and stability met the requirements of the guiding principles. After erdafitinib combined with itraconazole, the $\mathrm{C}_{\max }$ and $\mathrm{AUC}_{0 \rightarrow \mathrm{t}}$ of ripretinib increased by $38.35 \%$ and $36.36 \%$, respectively, and the $t_{1 / 2}$ was prolonged to $7.53 \mathrm{~h}$. After ripretinib combined with voriconazole, the $\mathrm{C}_{\max }$ and $\mathrm{AUC}_{0 \rightarrow \mathrm{t}}$ of ripretinib increased by $37.44 \%$ and $25.52 \%$, respectively, and the $t_{1 / 2}$ was prolonged to $7.33 \mathrm{~h}$.

Conclusion: A new and reliable UPLC-MS/MS technique was fully optimized and developed to detect the concentration of ripretinib in beagle dog plasma. Itraconazole and voriconazole could inhibit the metabolism of ripretinib in beagle dogs and increase the plasma exposure of ripretinib.

Keywords: itraconazole, voriconazole, ripretinib, UPLC-MS/MS, pharmacokinetics, beagle $\operatorname{dog}$

\section{Introduction}

Gastrointestinal stromal tumor (GISTs) originated from the interstitial cells of Cajal and were the most common sarcoma of the digestive tract. About $56 \%$ of GISTs occurred in the stomach and $32 \%$ in the small intestine, but they could arise in any part of the gastrointestinal tract. ${ }^{1,2}$ About $85-90 \%$ of the GIST cases were associated with known mutations. About $75-80 \%$ of GIST patients had mutations in the KIT receptor tyrosine kinase gene and $5-10 \%$ of GIST patients had mutations in the
Functional Experiment Teaching Center, School of Basic Medical Sciences, Henan University of Science and Technology, 263 Kaiyuan Avenue, Luoyang, 471023, People's Republic of China

Tel +8613698882699

Fax $+86379-64830346$

Email lyxiangjun@I26.com 
platelet-derived growth factor receptor alpha (PDGFRA) tyrosine kinase gene. Moreover, $10-15 \%$ of GIST patients had no mutations and were classified as wild type GIST. ${ }^{2,3}$

Recent advances in GIST treatment were very promising, and further trials and personalized treatment were needed within the context of multidisciplinary methods. ${ }^{4}$ Imatinib was a tyrosine kinase inhibitor (TKI) that targeted Brc-Abl (Philadelphia), KIT, and PDGFRA. Since the introduction of imatinib, the treatment of GIST patients with locally advanced and metastatic disease had improved dramatically. ${ }^{5}$ The traditional treatment of advanced GIST had taken imatinib as the first-line treatment, sunitinib as the second-line treatment and regorafenib as the third-line treatment. ${ }^{2}$ With the development of new molecular compounds such as ripretinib and avapritinib, the treatment of advanced GIST was developing rapidly. ${ }^{4}$ Demonstrated with the approval of ripretinib and avapritinib, the KIT and PDGFRA, as the oncogenic drivers of GIST, were still viable targets for therapy. ${ }^{1}$

Ripretinib (DCC-2618, Figure 1A) was designed to inhibit the full spectrum of mutant KIT and PDGFRA kinases which were found in cancers and myeloproliferative neoplasms, particularly in GISTs, and the heterogeneity of drugresistant KIT mutations in GISTs was a major challenge. ${ }^{6}$ Ripretinib was a novel switch-pocket inhibitor with broad activity against KIT and PDGFRA oncoproteins. Through a dual mechanism of action, ripretinib inhibited many of the most common primary and secondary activating KIT and PDGFRA mutants involved in GIST progression and ripretinib had become a new standard for the treatment of advanced and multi-drug resistant GIST patients. ${ }^{7,8}$ In May 2020, oral ripretinib received its first approval in the USA for the treatment of adult patients with advanced GIST who had received prior treatment with $\geq 3$ kinase inhibitors, including imatinib. ${ }^{9}$

The recommended dose of ripretinib was $150 \mathrm{mg}$ once daily, with or without food, until disease progression or unacceptable toxicity occurred. After oral administration of $150 \mathrm{mg}$ of ripretinib, the median time to reach $\mathrm{C}_{\max }$ for ripretinib was 4 hours and the time to reach a steady state was 14 days. ${ }^{9}$ After a single dose of ripretinib $150 \mathrm{mg}$, the mean $\mathrm{C}_{\max }, \mathrm{AUC}_{0-12 \mathrm{~h}}$ and $\mathrm{AUC}_{0-24 \mathrm{~h}}$ were $502 \mathrm{ng} / \mathrm{mL}$, $3773 \mathrm{ng} \cdot \mathrm{h} / \mathrm{mL}$ and $6634 \mathrm{ng} \cdot \mathrm{h} / \mathrm{mL}$ (59.8\%), respectively. However, ripretinib PK parameters after single doses were highly variable between patients, the coefficient of variation $(\mathrm{CV} \%)$ of mean $\mathrm{C}_{\max }, \mathrm{AUC}_{0-12 \mathrm{~h}}$ and $\mathrm{AUC}_{0-24 \mathrm{~h}}$ were $56.8 \%, 58.3 \%$ and $59.8 \%$, respectively. ${ }^{10}$

Ripretinib was mainly metabolised by cytochrome P450 (CYP) 3A4 and the main metabolite was DP-5439. Such inhibitors and inducers of CYP3A4 could affect the plasma concentrations of Ripretinib. Ripretinib and DP5439 could inhibit CYP2C8; therefore, they might affect other medicines that were cleared by CYP2C $8 .{ }^{11}$

Itraconazole and voriconazole were azole antifungals, which inhibit the 14- $\alpha$-demethylase and interfere with ergosterol biosynthesis in fungal cells, resulting in fungal death, and were the first choice for deep fungal infection in clinic. Itraconazole undergoes excessive hepatic metabolism involving CYP3A4 and the most active metabolite is hydroxyitraconazole. Voriconazole undergoes hepatic Phase I biotransformation involving CYP2C9, CYP2C19, and CYP3A4. Itraconazole is a strong inhibitor of CYP3A4,

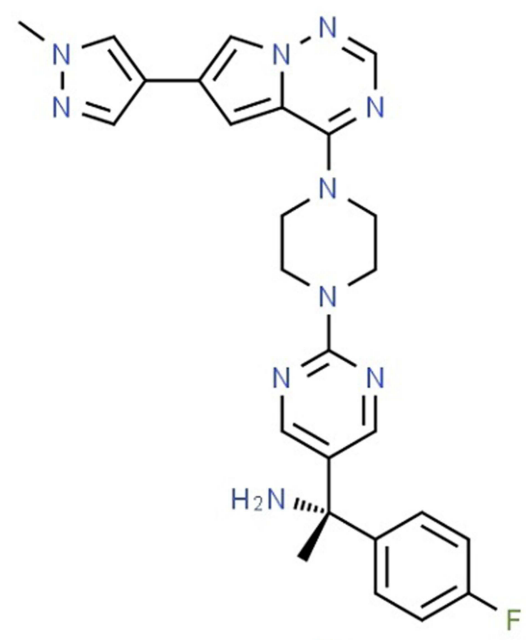

B 
voriconazole is a strong inhibitor of CYP2C19, CYP2C9 and a moderate inhibitor of CYP3A4. ${ }^{12}$ Azole antifungals competitively inhibit CYP3A4 to cause clinically significant drugdrug interactions (DDIs). ${ }^{13}$ The pharmacokinetic parameters of cyclophosphamide were increased by various extents when coadministered with fluconazole, itraconazole, or ketoconazole. Ketoconazole had the greatest effect on the PK of cyclophosphamide among the 3 triazole antifungals. ${ }^{14}$ Our previous research results show that posaconazole significantly increased the concentration of selinexor (a selective nuclear export inhibition) in rats, fluconazole, itraconazole, and isavuconazole have significant effects on pharmacokinetics of selinexor and increased plasma exposure of selinexor in rats. ${ }^{15,16}$

Invasive fungal infection was the main cause of incidence rate and mortality of cancer patients receiving intensive chemotherapy. ${ }^{17}$ Cancer patients, including GIST patients, may use antifungal drugs to control fungal infection, such as itraconazole and voriconazole. Hence, the possible DDIs should be paid attention to when ripretinib was combined with itraconazole or voriconazole. At present, there are no reports on the detection of plasma concentration of ripretinib. Therefore, it is very important to establish and develop a quantification method of ripretinib concentration for pharmacokinetics and DDI study. Consequently, in this study, we established an UPLC-MS/MS method to detect the plasma concentration of ripretinib in beagle dog plasma using avapritinib as the internal standard (IS, Figure 1B) and successfully used it for the pharmacokinetic effects of itraconazole and voriconazole on ripretinib in beagle dogs.

\section{Materials and Methods}

\section{Chemicals Materials}

Ripretinib (purity >98\%) was purchased from Shanghai Chuangshi Technology Company Limited. Avapritinib (IS, purity $>98 \%$ ) was purchased from Shanghai Chuangshi Technology Company Limited. HPLC pure methanol and acetonitrile were purchased from Tianjin kemio Chemical Reagent Co., Ltd. Analytical pure formic acid and sodium hydroxide were purchased from Tianjin No. 3 chemical reagent factory. Itraconazole capsules are produced by Xi'an Janssen Pharmaceutical Co., Ltd, Itraconazole capsules are produced by Sichuan Medco Huakang Pharmaceutical Co., Ltd.

\section{Instruments}

The UPLC instrument was Waters ACQUITY H-Class system which consisted of the following: Quaternary solvent manager, Sample manager-flow through needle, High temperature column heater with active pre-heating. The mass spectrometry was Waters Xevo TQ-S triple quadrupole tandem mass spectrometer, and the ionization source was electrospray ionization source (ESI source).

\section{Standard Solutions Ready}

$10 \mathrm{mg}$ of ripretinib reference substance was accurately weighted in a $10 \mathrm{~mL}$ volumetric flask and dissolved it with methanol to $10 \mathrm{~mL}$ to obtain the ripretinib standard stock solution with a concentration of $1 \mathrm{mg} / \mathrm{mL}$. Concentrations of $100 \mu \mathrm{g} / \mathrm{mL}, 10 \mu \mathrm{g} / \mathrm{mL}, 1 \mu \mathrm{g} / \mathrm{mL}$ standard application solutions were diluted to the standard stock solution with methanol (diluted temporarily). The IS stock solution was prepared in the same way and diluted it with methanol to $2.5 \mu \mathrm{g} / \mathrm{mL}$ IS application solution. All the solutions were stored in a refrigerator at $-4^{\circ} \mathrm{C}$.

\section{Analytical Conditions}

The chromatographic column was an ACQUITY UPLC $\mathrm{BEH}$ C18 column $(2.1 \mathrm{~mm} \times 50 \mathrm{~mm}, 1.7 \mathrm{um})$, and the mobile phase consisted of $0.1 \%$ formic acid and acetonitrile. The gradient elution program is listed in Table 1. The flow rate was set at $0.40 \mathrm{~mL} / \mathrm{min}$, the column temperature was $45^{\circ} \mathrm{C}$, and the analysis time was $2.0 \mathrm{~min}$.

Under the positive ion condition of ESI source, mass spectrometry was carried out in multiple reaction monitoring mode (MRM). The parent ion and daughter ion used for the quantification of ripretinib and IS were as follows: $\mathrm{m} / \mathrm{z}$ 509.93-416.85 for ripretinib and $\mathrm{m} / \mathrm{z} 499.1$ 482.09 for IS, respectively. The cone voltage of ripretinib and IS were $30 \mathrm{~V}$ and $30 \mathrm{~V}$, and the collision voltage was $25 \mathrm{~V}$ and $10 \mathrm{~V}$, respectively.

\section{Sample Preparation}

$100 \mu \mathrm{L}$ of beagle dog plasma to be tested was taken into a $2.0 \mathrm{~mL}$ EP tube, $20 \mu \mathrm{L}$ of IS working solution was

Table I The Gradient Elution Program of UPLC

\begin{tabular}{|l|c|c|c|}
\hline $\begin{array}{l}\text { Time } \\
(\mathbf{m i n})\end{array}$ & $\begin{array}{c}\text { 0.1\% Formic } \\
\text { Acid }\end{array}$ & Acetonitrile & $\begin{array}{c}\text { Flow Rate (mL/ } \\
\mathbf{m i n})\end{array}$ \\
\hline 0 & $70 \%$ & $30 \%$ & 0.4 \\
\hline 0.9 & $15 \%$ & $85 \%$ & 0.4 \\
\hline 1.0 & $70 \%$ & $30 \%$ & 0.4 \\
\hline 2.0 & $70 \%$ & $30 \%$ & 0.4 \\
\hline
\end{tabular}


added, and mixed well. $200 \mu \mathrm{L}$ of $1 \mathrm{~mol} / \mathrm{L}$ sodium hydroxide solution was added and mixed well. Then, $1 \mathrm{~mL}$ of ethyl acetate was added and vortexed for $1 \mathrm{~min}$. The upper organic phase was taken into another $2.0 \mathrm{~mL}$ EP tube and blowed dry with nitrogen flow. The residue was dissolved with $100 \mu \mathrm{L}$ mobile phase, and $50 \mu \mathrm{L}$ supernatant was taken into the sample bottle of an automatic injector. The injection volume was $2 \mu \mathrm{L}$.

\section{Method Validation}

The main purpose of analytical method validation was to prove the reliability of a specific method for determining the concentration of analytes in a biological matrix. The main characteristics of a bioanalytical method include: selectivity, lower limit of quantification (LLOQ), standard curve, accuracy, precision, matrix effect, stability of analytes in biological matrix and in the whole process of storage and treatment in solution. So, the specificity, precision and accuracy, standard curve and LLOQ, recovery, matrix effect and sample stability of the method were verified according to the guiding principles of FDA and CFDA. ${ }^{18,19}$

\section{Animal Experiments}

Six beagle dogs, half male and half female, weighing 6.0-8.0 kg, were purchased from Hubei Yizhicheng Biotechnology Co., Ltd., the production license No.: SCXK(E) 2016-0020. The beagle dogs were raised in the experimental animal center of Henan University of Science and Technology. The animal experiment was approved by the animal ethics committee of Henan University of Science and Technology. During the experiment, the operating procedures of experimental animals were strictly followed and the welfare of animals was guaranteed, and the name of the guidelines followed for the welfare and treatment of the laboratory animals was the Laboratory animals guidelines for ethical review of welfare (GB/T 35892-2018, General Administration of Quality Supervision, Inspection and Quarantine of the People's Republic of China, Standardization Administration of the People's Republic of China).

This animal experiment adopted a three period selfcontrol experimental design. All the beagle dogs fasted for 12 hours before the experiment but were free to drink water. In the first period (Group A), ripretinib was dissolved in $0.5 \%$ carboxymethyl cellulose sodium (CMC-Na) solution, and was orally administered to beagle dogs at a dose of
$5 \mathrm{mg} / \mathrm{kg}$. The blank blood was collected before erdafitinib was given, then at the different time points of $0.33,0.67,1$, $1.5,2,3,4,6,8,12,24$, and $48 \mathrm{~h}$, blood samples (approximately $1.0 \mathrm{~mL}$ ) were collected from the veins of anterior and posterior limbs and taken into $1.5 \mathrm{~mL}$ heparinized EP tubes. The plasma were separated by centrifugation for 10 minutes at $10,000 \mathrm{rpm}$, and frozen at $-20^{\circ} \mathrm{C}$ for later analysis.

After a week of drug wash out period, the second period (Group B) of experiment was carried out. On the day of the experiment, the same six beagle dogs were orally given itraconazole at a dose of $7 \mathrm{mg} / \mathrm{kg}$ (after the shell of itraconazole capsule was removed, it was dissolved in $0.1 \%$ sodium carboxymethyl cellulose solution with a concentration of $14 \mathrm{mg} / \mathrm{mL}$ ), after $30 \mathrm{~min}, 5 \mathrm{mg} / \mathrm{kg}$ ripretinib was orally administered to beagle dogs. The time point of blood collection was the same as that of the first period.

Then, after a week of the second drug wash out period, the third period (Group C) of experiment was carried out. On the day of the experiment, the same six beagle dogs were orally given voriconazole at a dose of $7 \mathrm{mg} / \mathrm{kg}$ (after the shell of voriconazole capsule was removed, it was dissolved in $0.1 \%$ sodium carboxymethyl cellulose solution with a concentration of $14 \mathrm{mg} / \mathrm{mL}$ ), after $30 \mathrm{~min}$, ripretinib was orally administered at a dose of $5 \mathrm{mg} / \mathrm{kg}$. The time point of blood collection was the same as that of the first period.

\section{Pharmacokinetics Study}

The ripretinib concentrations in beagle dog plasma were detected by batch processing according to the established UPLC-MS/MS method. The analytical batch included accompanying standard curves and quality control samples. Then, DAS (Drug and Statistics, version 2.0) were used to calculate the important pharmacokinetic parameters of erdafitinib through the statistical moment method. The main pharmacokinetic parameters of erdafitinib were as follows: $T_{\max }, C_{\max }, t_{1 / 2}, C L, V d$, and AUC. Then, the mean concentration-time curve of ripretinib was drawn in groups $\mathrm{A}, \mathrm{B}$ and $\mathrm{C}$.

\section{Statistical Analysis}

The data were processed using SPSS 18.0 statistical software. The group A was taken as the control group, and the differences of pharmacokinetic parameters between group B and group A, between group $\mathrm{C}$ and group A were compared, respectively. The $P$ value was calculated by independent sample $t$-test, $P<0.05$, which was statistically significant. 


\section{Results and Discussion \\ Method Development and Optimization}

UPLC-MS/MS technique has the advantages of high sensitivity, strong specificity, short analysis time and good reproducibility. Therefore, it is often used in the detection of biological samples, the study of pharmacokinetics and DDI. ${ }^{15,20,21}$

Mobile phases such as methanol-water, acetonitrilewater and $0.1 \%$ formic acid-acetonitrile were investigated. It was found that each component showed a high detection sensitivity and good peak shape when $0.1 \%$ formic acidacetonitrile was used as the mobile phase. At the same time, the gradient elution procedure could greatly improve the separation speed and efficiency, and the effective separation could be realized within 2 min.

In this experiment, the positive and negative ion modes were used to investigate the response value of ripretinib and IS. The results showed that ripretinib and IS were more responsive in positive ion mode. Under the positive ion mode, the parent ion and daughter ion of ripretinib were $\mathrm{m} /$ z $503.93 \rightarrow 416.85$, respectively, the parent ion and daughter ion of IS were $\mathrm{m} / \mathrm{z} 499.10 \rightarrow 482.09$. The figures of MRM ion transitions from parent ion to daughter ion of ripretinib and IS are shown in Figure 2. At the same time, the conditions of spray voltage, air curtain gas, atomizing gas, auxiliary heating gas and auxiliary gas temperature were optimized.

\section{Selectivity}

The selectivity was demonstrated by the blank plasma from 6 beagle dogs, and the blank plasma were analyzed and evaluated for interference. The chromatograms of blank beagle dog plasma, ripretinib plasma standard and beagle dog sample after administration are shown in Figure 3. It could be seen from the figures that ripretinib and IS were well separated, the endogenous substances did not interfere with the detection, and the retention times of ripretinib and IS were $0.84 \mathrm{~min}$ and $0.63 \mathrm{~min}$, respectively.

Chromatographic separation was based on the difference of adsorption and solubility of analytes by stationary phase. The components with strong adsorption or solubility had large partition coefficient and long retention time; On the contrary, the components with weak adsorption or solubility had small partition coefficient and short retention time. It could be seen from the chromatogram that the retention time of ripretinib was slightly longer than that of IS. Therefore, under this chromatographic condition, the partition coefficient of ripretinib was slightly larger than that of IS.

\section{Linearity and LLOQ}

The ripretinib standard working solutions with different concentrations and volumes were added successively into eight EP tubes $(2.0 \mathrm{~mL})$, and then the blank beagle dog plasma with different volumes was added to prepare plasma samples with concentrations equivalent to 1,5 , $10,50,100,250,500$ and $1000 \mathrm{ng} / \mathrm{mL}$. After treatment according to sample preparation, the samples were analyzed, and the peak area of ripretinib (As) and the peak area of IS (Ai) were recorded. As/Ai was taken as the ordinate $\mathrm{y}$ and the corresponding point concentration was taken as the abscissa $\mathrm{x}$, the standard curve was drawn. The weighted least square method was used for standard curve
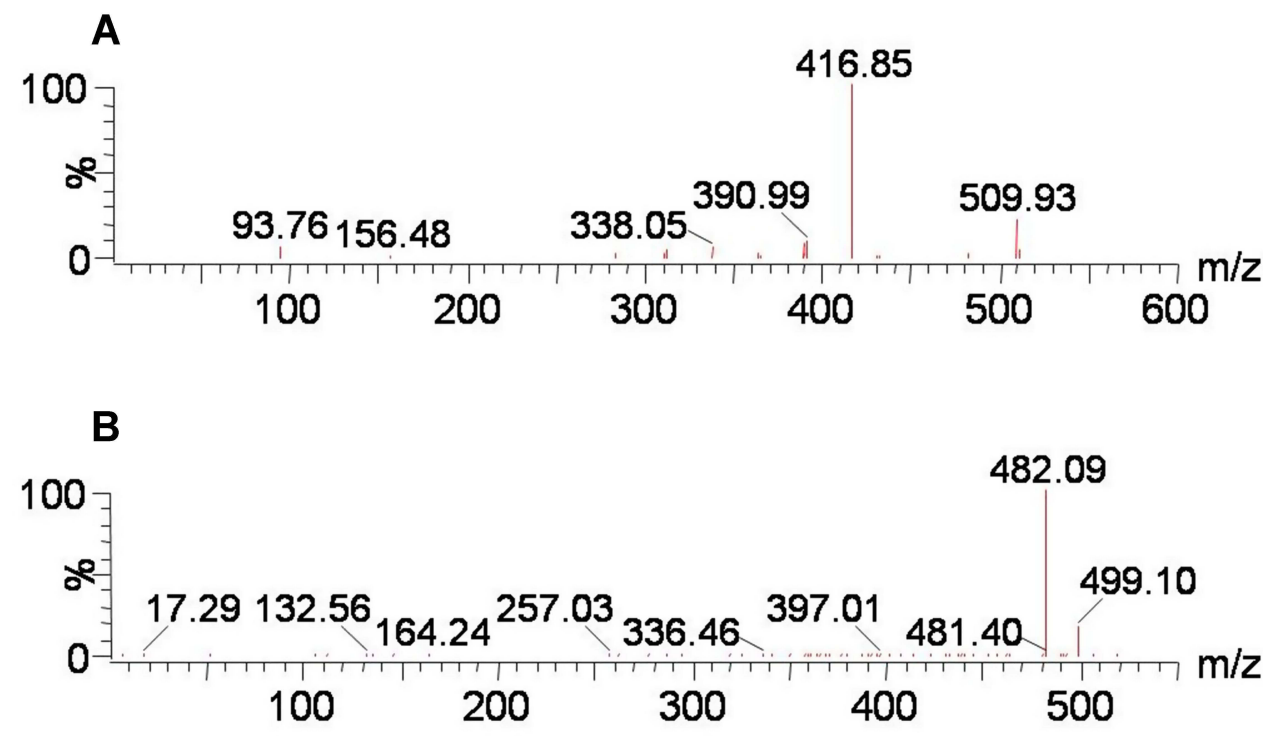

Figure 2 The ion transitions from parent ion to daughter ion of ripretinib (A) and IS (B). 
A
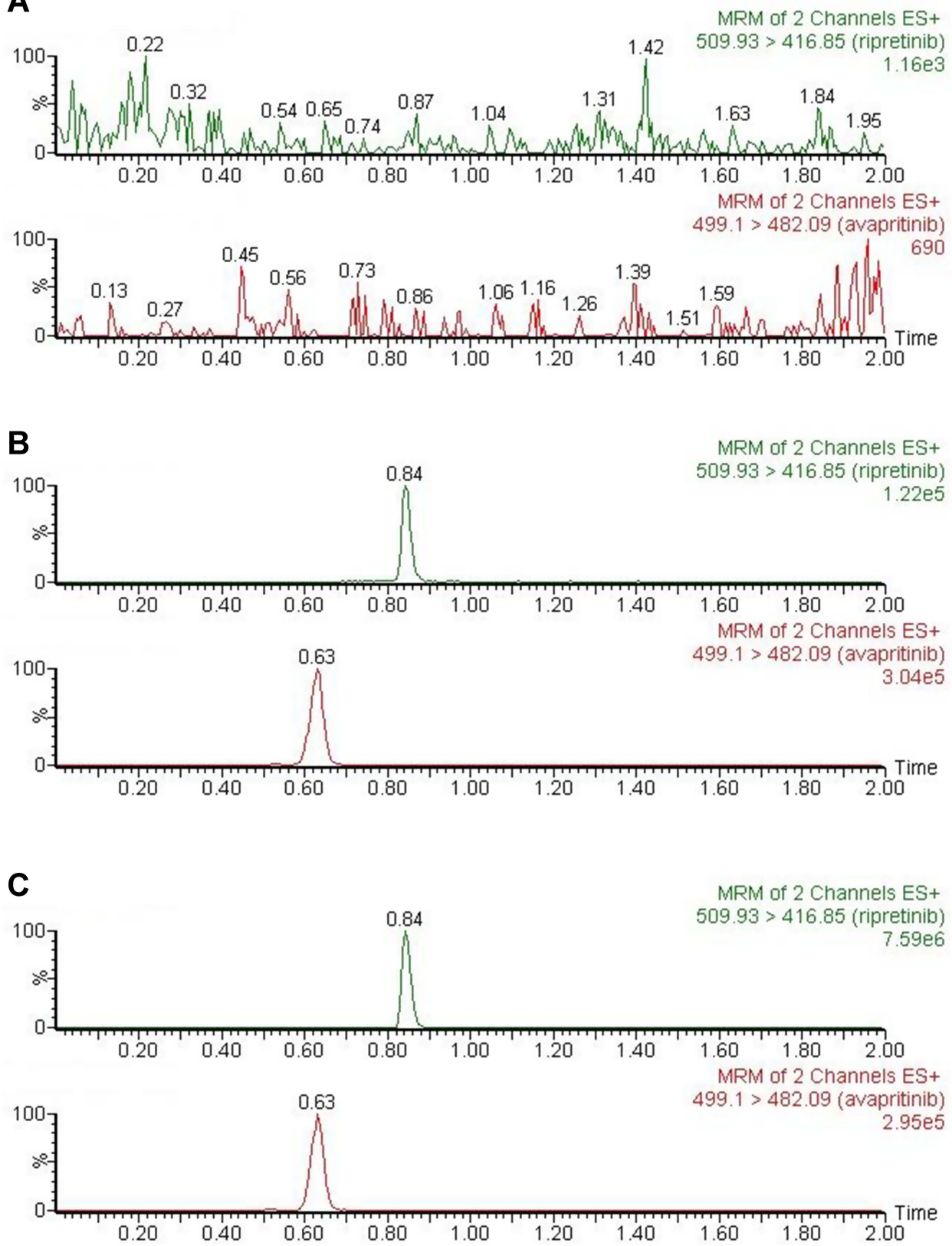

Figure 3 Representative chromatograms of ripretinib and IS. (A) blank beagle dog plasma; (B) blank plasma spiked with standard solutions; (C) sample obtained from beagle dog at $2.0 \mathrm{~h}$ after oral administration of $5 \mathrm{mg} / \mathrm{kg}$ ripretinib.

regression, and the weight was $1 / \mathrm{x}^{2}$. The regression equation of the standard curve of ripretinib was $\mathrm{y}=3.90 \times 10^{-3}$ $\mathrm{x}+4.37 \times 10^{-2}\left(\mathrm{R}^{2}=0.9995\right)$. The LLOQ for this experiment was the lowest concentration of the standard curve $(1 \mathrm{ng} / \mathrm{mL})$.

\section{Precision and Accuracy}

The low, medium and high concentrations $(2.5,100,750$ $\mathrm{ng} / \mathrm{mL}$ ) of ripretinib plasma standard solution were prepared with 6 copies of each concentration, and then were treated according to the plasma sample preparation method. Each concentration was measured on the same day, and the intra-day precision was calculated. The same measurement was carried out for 3 consecutive days to calculate the inter-day precision. The precision was expressed by relative standard deviation (RSD \%) and the accuracy was expressed by relative error (RE \%). The intra-day and inter-day precision and accuracy of ripretinib are shown in Table 2. The precision (\% RSD) did not exceed $8.77 \%$, the accuracy (\% RE) was in the range 
from $-2.80 \%$ to $1.06 \%$ at low, medium and high concentrations and met the requirements of validation.

\section{Recovery and Matrix Effect}

The low, medium and high concentrations $(2.5,100,750 \mathrm{ng} /$ $\mathrm{mL}$ ) of ripretinib plasma standard solution were prepared with 6 copies of each concentration, and then were treated according to the plasma sample preparation method, the samples were tested, and the peak area of ripretinib was recorded as A. Then, the pure standard solution of the corresponding concentration of ripretinib was prepared and was detected directly, and the peak area of ripretinib was recorded as B. The absolute recovery was calculated by $\mathrm{A} /$ B*100\%. The matrix effect of ripretinib was determined by calculating the ratio of the peak area in the presence of the matrix (ripretinib and IS were added after extraction from the blank matrix) to the corresponding peak area without the matrix. The recovery and the matrix effect of ripretinib at concentrations of $2.5,100$, and $750 \mathrm{ng} / \mathrm{mL}$ are presented in
Table 3. The results indicated that the absolute recovery was higher than $85 \%$ and the matrix effect did not affect the determination of ripretinib.

\section{Stability}

The low, medium and high concentrations $(2.5,100,750 \mathrm{ng} /$ $\mathrm{mL}$ ) of ripretinib plasma standard solution were prepared with 6 copies of each concentration. The stability of plasma samples stored at room temperature and frozen storage, the stability of plasma samples under freeze-thaw conditions, and the stability of treated plasma samples in automatic sampler were investigated. The results are presented in Table 4 which showed that ripretinib had good stability under four conditions, and the RSD was less than $6.04 \%$.

\section{Pharmacokinetics Effects}

Figure 4A displays the average plasma drug concentrationtime curves of ripretinib in Group $\mathrm{A}$ and $\mathrm{B}$, the average plasma concentration-time curves of ripretinib in Group

Table 2 Precision and Accuracy of Ripretinib in Beagle Dog Plasma $(n=6)$

\begin{tabular}{|l|c|c|c|c|c|c|}
\hline \multirow{2}{*}{ Added (ng/mL) } & \multicolumn{3}{|c|}{ Intra-Day } & \multicolumn{3}{c|}{ Inter-Day } \\
\cline { 2 - 8 } & Found (ng/mL) & RSD (\%) & RE (\%) & Found (ng/mL) & RSD (\%) & RE (\%) \\
\hline 1.0 & $0.99 \pm 0.09$ & 8.77 & -0.65 & $1.01 \pm 0.07$ & 7.28 & 1.06 \\
\hline 2.5 & $2.43 \pm 0.16$ & 6.48 & -2.80 & $2.51 \pm 0.07$ & 2.64 & 0.47 \\
\hline 100 & $100.24 \pm 4.55$ & 4.54 & 0.24 & $99.37 \pm 1.05$ & 1.06 & -0.63 \\
\hline 750 & $753.11 \pm 15.37$ & 2.04 & 0.41 & $735.91 \pm 14.90$ & 2.02 & -1.88 \\
\hline
\end{tabular}

Table 3 Recovery and Matrix Effect of Ripretinib in Beagle Dog Plasma $(n=6$, Mean \pm SD)

\begin{tabular}{|l|c|c|c|c|}
\hline Skipped (ng/mL) & Recovery (\%) & RSD (\%) & Matrix Effect (\%) & RSD (\%) \\
\hline 2.5 & $85.39 \pm 2.97$ & 3.48 & $96.64 \pm 6.11$ & 6.32 \\
\hline 100 & $86.55 \pm 3.37$ & 3.89 & $102.85 \pm 3.17$ & 3.08 \\
\hline 750 & $87.15 \pm 2.88$ & 3.31 & $100.06 \pm 2.79$ & 2.79 \\
\hline
\end{tabular}

Table 4 Stability Results of Ripretinib in Beagle Dog Plasma Under Four Conditions $(n=6)$

\begin{tabular}{|l|c|c|c|c|c|c|c|c|}
\hline \multirow{2}{*}{$\begin{array}{l}\text { Added } \\
(\mathbf{n g} / \mathbf{m L} \text { ) }\end{array}$} & \multicolumn{2}{|c|}{ Room Temperature, 4 h } & \multicolumn{2}{|c|}{ Autosampler 4 ${ }^{\circ} \mathbf{C}, \mathbf{6} \mathbf{~ h}$} & \multicolumn{2}{|c|}{ Three Freeze-Thaw } & \multicolumn{2}{|c|}{$-20^{\circ} \mathbf{C}, 4$ Weeks } \\
\cline { 2 - 10 } & RSD (\%) & RE (\%) & RSD (\%) & RE (\%) & RSD (\%) & RE (\%) & RSD (\%) & RE (\%) \\
\hline 2.5 & 6.07 & 1.07 & 5.91 & -1.00 & 5.46 & -5.93 & 6.04 & 1.87 \\
\hline 100 & 3.26 & -1.93 & 4.08 & 2.03 & 4.89 & 1.05 & 2.82 & -3.55 \\
\hline 750 & 3.28 & 0.76 & 2.44 & -4.42 & 3.05 & 1.26 & 2.20 & -4.55 \\
\hline
\end{tabular}



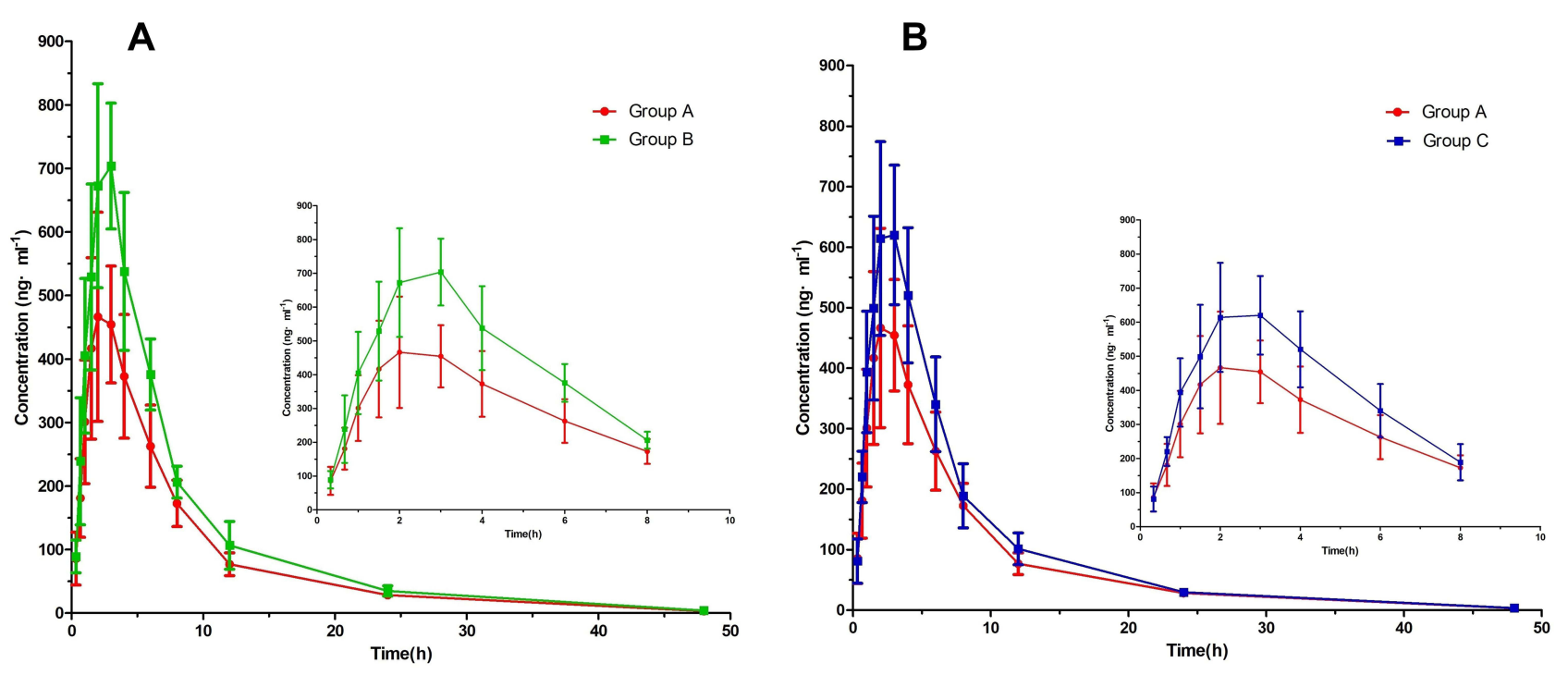

Figure 4 (A, B) Mean plasma concentration-time curves of ripretinib after oral administration of ripretinib at a single dose of $5 \mathrm{mg} / \mathrm{kg}$ in group A, B and C ( $=6$ ).

$\mathrm{A}$ and $\mathrm{C}$ are displayed in Figure 4B. The main parameters of pharmacokinetics of ripretinib in Groups $\mathrm{A}, \mathrm{B}$ and $\mathrm{C}$ are summarized in Table 5.

The results of the study showed that after itraconazole combined with ripretinib Voriconazole the $\mathrm{C}_{\max }$ and $\mathrm{AUC}_{0 \rightarrow \mathrm{t}}$ of ripretinib increased by $38.35 \%$ and $36.35 \%$, respectively, $\mathrm{V}$ and $\mathrm{CL}$ also were decreased accordingly and the $t_{1 / 2}$ was prolonged to $7.53 \mathrm{~h}$. After voriconazole combined with ripretinib, the $\mathrm{C}_{\max }$ and $\mathrm{AUC}_{0 \rightarrow \mathrm{t}}$ of ripretinib increased by $27.44 \%$ and $25.52 \%$, respectively, $\mathrm{V}$ and $\mathrm{CL}$ also were decreased accordingly, and the $t_{1 / 2}$ was prolonged to $7.33 \mathrm{~h}$. It is

Table 5 The Main Pharmacokinetic Parameters of Ripretinib After Oral Administration of Ripretinib at a Single Dose of $5 \mathrm{mg} / \mathrm{kg}$ in Group A, B and C $(n=6$, Mean \pm SD)

\begin{tabular}{|l|c|c|c|}
\hline Parameters & Group A & Group B & Group C \\
\hline$T_{\max }(\mathrm{h})$ & $2.25 \pm 0.61$ & $2.67 \pm 0.52$ & $2.50 \pm 0.55$ \\
\hline $\mathrm{C}_{\max }(\mathrm{ng} / \mathrm{mL})$ & $543.85 \pm 109.02$ & $752.43 \pm 97.08^{*}$ & $692.19 \pm 104.62^{*}$ \\
\hline $\mathrm{t}_{\mathrm{l} / 2}(\mathrm{~h})$ & $6.96 \pm 0.31$ & $7.53 \pm 0.83$ & $7.33 \pm 1.27$ \\
\hline $\mathrm{CLz} / \mathrm{F}(\mathrm{L} / \mathrm{h} / \mathrm{kg})$ & $1.26 \pm 0.16$ & $0.93 \pm 0.16$ & $1.01 \pm 0.14$ \\
\hline $\mathrm{Vz} / \mathrm{F}(\mathrm{L} / \mathrm{kg})$ & $12.65 \pm 1.75$ & $10.26 \pm 2.78$ & $10.70 \pm 2.73$ \\
\hline $\begin{array}{l}\mathrm{AUC} \\
(\mathrm{ng} \cdot \mathrm{h} / \mathrm{mL})\end{array}$ & $3998.15 \pm 556.19$ & $5451.64 \pm 994.02^{*}$ & $5018.56 \pm 701.04^{*}$ \\
\hline $\begin{array}{l}\mathrm{AUC} \mathrm{C}_{0 \rightarrow \infty} \\
(\mathrm{ng} \cdot \mathrm{h} / \mathrm{mL})\end{array}$ & $4030.67 \pm 563.22$ & $5494.42 \pm 997.98^{*}$ & $5056.46 \pm 698.13^{*}$ \\
\hline
\end{tabular}

Notes: Group A - ripretinib, Group B - itraconazole + ripretinib, Group C voriconazole + ripretinib. *Compared with group A, the difference was statistically significant $(P<0.05)$. suggested that itraconazole and voriconazole could effect the pharmacokinetics of ripretinib in beagle dogs and increase the plasma exposure of erdafitinib. Therefore, when ripretinib was combined with itraconazole and voriconazole, possible pharmacokinetic DDIs should be considered.

\section{Conclusions}

In this study, a reliable, rapid and specific UPLC-MS /MS method was fully optimized and firstly developed to detect the plasma concentration of ripretinib concentration in beagle dog plasma. The optimized method had the advantage of short analysis time $(2.0 \mathrm{~min})$, excellent linearity (1-1000 ng/mL), high recovery, good precision and strong specificity. Itraconazole and voriconazole could effect the pharmacokinetics of ripretinib in beagle dogs and increase the plasma exposure of erdafitinib. Therefore, when ripretinib was combined with itraconazole and voriconazole, possible pharmacokinetic DDIs should be considered.

\section{Author Contributions}

All authors made a significant contribution to the work reported, whether that is in the conception, study design, execution, acquisition of data, analysis and interpretation, or in all these areas; took part in drafting, revising or critically reviewing the article; gave final approval of the version to be published; have agreed on the journal to which the article has been submitted; and agree to be accountable for all aspects of the work. 


\section{Disclosure}

The authors declare that they have no known competing financial interests or personal relationships that could have appeared to influence the work reported in this paper.

\section{References}

1. Bauer S, George S, von Mehren M, Heinrich MC. Early and next-generation KIT/PDGFRA kinase inhibitors and the future of treatment for advanced gastrointestinal stromal tumor. Front Oncol. 2021;11:672500. doi:10.3389/fonc.2021.672500

2. Patel SR, Reichardt P. An updated review of the treatment landscape for advanced gastrointestinal stromal tumors. Cancer. 2021;127 (13):2187-2195. doi:10.1002/cncr.33630

3. Vallilas C, Sarantis P, Kyriazoglou A, et al. Gastrointestinal Stromal Tumors (GISTs): novel therapeutic strategies with immunotherapy and small molecules. Int J Mol Sci. 2021;22(2):493. doi:10.3390/ ijms 22020493

4. Farag S, Smith MJ, Fotiadis N, Constantinidou A, Jones RL. Revolutions in treatment options in gastrointestinal stromal tumours (GISTs): the latest updates. Curr Treat Options Oncol. 2020;21(7):55. doi:10.1007/s11864-020-00754-8

5. Blay JY. A decade of tyrosine kinase inhibitor therapy: historical and current perspectives on targeted therapy for GIST. Cancer Treat Rev. 2011;37(5):373-384. doi:10.1016/j.ctrv.2010.11.003

6. Smith BD, Kaufman MD, Lu WP, et al. Ripretinib (DCC-2618) is a switch control kinase inhibitor of a broad spectrum of oncogenic and drug-resistant KIT and PDGFRA variants. Cancer Cell. 2019;35 (5):738-751. doi:10.1016/j.ccell.2019.04.006

7. Lostes-Bardaji MJ, García-Illescas D, Valverde C, Serrano C. Ripretinib in gastrointestinal stromal tumor: the long-awaited step forward. Ther Adv Med Oncol. 2021;13:1-12. doi:10.1177/ 1758835920986498

8. Zalcberg JR. Ripretinib for the treatment of advanced gastrointestinal stromal tumor. Therap Adv Gastroenterol. 2021;14:1-12. doi: $10.1177 / 17562848211008177$

9. Dhillon S. Ripretinib: first Approval. Drugs. 2020;80(11):1133-1138. doi:10.1007/s40265-020-01348-2

10. Janku F, Abdul Razak AR, Chi P, et al. Switch control inhibition of KIT and PDGFRA in patients with advanced gastrointestinal stromal tumor: a Phase I study of ripretinib. J Clin Oncol. 2020;38 (28):3294-3303. doi:10.1200/JCO.20.00522

11. Falconer N, Ryan M, Badman B, Carrington C. Ripretinib for gastrointestinal stromal tumours. Aust Prescr. 2021;44:112. doi:10.18773/ austprescr.2021.025
12. Bellmann R, Smuszkiewicz P. Pharmacokinetics of antifungal drugs: practical implications for optimized treatment of patients. Infection 2017;45(6):737-779. doi:10.1007/s15010-017-1042-z

13. Yamaguchi Y, Akiyoshi T, Kawamura G, et al. Comparison of the inhibitory effects of azole antifungals on cytochrome P450 3A4 genetic variants. Drug Metab Pharmacokinet. 2021;38:100384. doi:10.1016/j.dmpk.2021.100384

14. Cai T, Liao Y, Chen Z, Zhu Y, Qiu X. The influence of different triazole antifungal agents on the pharmacokinetics of cyclophosphamide. Ann Pharmacother. 2020;54(7):676-683. doi:10.1177/1060028019896894

15. Zhou CJ, Wang HJ, Zhou CY, Li CF, Zhu MJ, Qiu XJ. Establishment and verification of UPLC-MS/MS technique for pharmacokinetic drug-drug interactions of selinexor with posaconazole in rats. Drug Des Devel Ther. 2021;15:1561-1568. doi:10.2147/DDDT.S303928

16. Li SL, Zhang Y, Cheng QS, Xin JZ, Dong ZQ, Qiu XJ. UPLC-MS /MS measurement of the effect of isavuconazole, itraconazole and fluconazole on the pharmacokinetics of selinexor in rats. Infect Drug Resist. 2020;13:3153-3161. doi:10.2147/IDR.S269831

17. Mousset S, Buchheidt D, Heinz W, et al. Treatment of invasive fungal infections in cancer patients-updated recommendations of the Infectious Diseases Working Party (AGIHO) of the German Society of Hematology and Oncology (DGHO). Ann Hematol. 2014;93 (1):13-32. doi:10.1007/s00277-013-1867-1

18. US Food and Drug Administration. Guidance for industry: bioanalytical method validation. Rockville, MD, USA: US Department of Health and Human Services, US FDA, Center for Drug Evaluation and Research; 2018. Available from: https://www.fda.gov/regulator yinformation/search-fda-guidance-documents/bioanalyticalmethodva lidation-guidance-industry. Accessed August 10, 2018.

19. Chinese Pharmacopoeia Commission. Guidelines for Validation of Quantitative Analysis Methods for Biological Samples. Pharmacopoeia of the People's Republic of China. China Medical Science and Technology Press; Vol. II, 2020:466-472.

20. Tang C, Niu X, Shi L, Zhu H, Lin G, Xu RA. In vivo pharmacokinetic drug-drug interaction studies between fedratinib and antifungal agents based on a newly developed and validated UPLC/MS-MS method. Front Pharmacol. 2021;11:626897. doi:10.3389/ fphar.2020.626897

21. Hu J, Su XJ, Si HL, et al. Simultaneous determination of celecoxib, dezocine and dexmedetomidine in beagle plasma using UPLC-MS/ MS method and the application in pharmacokinetics. Drug Des Devel Ther. 2021;15:2529-2541. doi:10.2147/DDDT.S314562
Drug Design, Development and Therapy

\section{Publish your work in this journal}

Drug Design, Development and Therapy is an international, peerreviewed open-access journal that spans the spectrum of drug design and development through to clinical applications. Clinical outcomes, patient safety, and programs for the development and effective, safe, and sustained use of medicines are a feature of the journal, which has also been accepted for indexing on PubMed Central. The manuscript management system is completely online and includes a very quick and fair peer-review system, which is all easy to use. Visit http://www. dovepress.com/testimonials.php to read real quotes from published authors. 